\title{
Simulation Research on Mechanical Properties and Crack Growth of Tobacco Leaf
}

\author{
Ruidong $\mathrm{LI}^{\mathrm{a}, 1}$, Keqiang $\mathrm{LI}^{\mathrm{a}}$, Wenyong HUANG ${ }^{\mathrm{a}}$, Zehui YANG ${ }^{\mathrm{a}}$, Xiaobing ZHANG ${ }^{\mathrm{b}}$ \\ and Jianguo LIU ${ }^{\mathrm{b}}$ \\ a Yunnan Leaf Tobacco Redrying Co. Ltd., Kunming 650031, China \\ ${ }^{\mathrm{b}}$ China Tobacco Zhejiang Industrial Co., Ltd., Huangzhou 310024, China
}

\begin{abstract}
Aimed at the problem of crack propagation of tobacco leaves subjected to (type I) tensile force under specific conditions. This paper mainly discusses whether tobacco stem can inhibit the crack propagation. After temperature and humidity treatment of the selected tobacco samples, an electronic universal testing machine was used to conduct fracture tensile test of tobacco leaves. Through the simulation analysis of the type I crack propagation of tobacco slices, sections of tobacco stems and whole tobacco leaves, the graphs of force variation with time and stress-strain curves were obtained. The inhibiting effect of tobacco stem on crack was verified by comparison and analysis. The results showed that the tobacco stem did inhibit the crack propagation in the process of crack propagation.
\end{abstract}

Keywords. Crack propagation, simulation analysis, tensile fracture, temperature and humidity treatment, stress and strain.

\section{Introduction}

Tobacco threshing process is a complicated, time-varying process with randomness, uncertainty, and highly nonlinear characteristics. The structure of sheet tobacco is an important index to measure the quality of threshing leaves[1], The three main factors that affect the quality of the leaves are the physical characteristics of the leaves, the equipment performance and the technological parameters of the threshing process[2]. Li Yuefeng, et al[3] conducted experiments on the influence of different temperature and humidity on the quality of threshing, and the results showed that increasing the temperature and moisture content of the original tobacco appropriately before threshing is beneficial to improve the threshing quality. Luo Fuwei, et al[4] concluded through experiments that the tensile strength of tobacco leaves increased with the increase of moisture content before the moisture content was $18 \%$, reached the maximum value when the moisture content was $18 \%$, and then gradually deteriorated with the increase of moisture content. The temperature of tobacco leaves mainly affects the flexibility of tobacco leaves. Low temperature increases the brittleness and causes serious crushing during threshing. At the same time, it will increase the number of leaves in the stem after

${ }^{1}$ Corresponding Author, Ruidong LI, Yunnan Leaf Tobacco Redrying Co. Ltd., Kunming 650031, China; Email:371856443@qq.com. 
leaf beating and reduce the percentage of pieces; When the temperature is too high, the threshing efficiency will also be affected, and the repeated threshing rate will be low and the material will be easily blocked; The final analysis compares that the breaking rate of tobacco leaves is highest when the temperature of tobacco leaves is around $50^{\circ} \mathrm{C}$. Many scholars have conducted research on the threshing stage, but they are all based on experiments, and the conclusions of each experiment are different. There is no in-depth study on the internal mechanism of the threshing process and the constitutive model of tobacco. This paper discusses the constitutive model of tobacco leaf, analyzes and studies the problem of tobacco leaf breakage in the process of threshing. From 1999 Belytschk [5] and Moes [6] proposed the term extended finite element method (XFEM)[7]. Through some special configurations with cracks, scholars have obtained the closedform solutions of stress intensity factors. Those solutions of these stress intensity factors have been compiled into a stress intensity factor manual for engineers' reference [8-10], the rapid development and wide application of the extended finite element method are closely related to its own advantages [11]. This paper mainly studies the mechanical properties of the constitutive model of tobacco leaves at a temperature of $50^{\circ} \mathrm{C}$ and a moisture content of about $18 \%$, XFEM in abaqus is used as the main tool to study the fracture problem of tobacco leaves, the crack cracking, propagation and crack arrest under the action of dynamic load, static load, temperature and corrosion of medium are studied [12].

In this paper, the stress-strain curves of tobacco leaves and tobacco stems are obtained by doing specific mechanical characteristics tests of tobacco leaves and tobacco stems. With the help of XFEM method, the finite element model of tobacco leaves and tobacco stems is established in the simulation and the corresponding mechanical tests are carried out. The validity of the model is verified by comparing the stress-strain curves of simulation and actual experiment.

\section{Tobacco Leaves Breaking Tensile Test}

\subsection{Test Equipment and Materials}

The material is tobacco leaves which up to standard after moisture regaining treatment, the temperature is $50^{\circ} \mathrm{C}$, the moisture content is about $18 \%$, the equipment used is an electronic universal tensile testing machine and a self-made tobacco leaf vein clamping device, etc. The software used for the test is MaterialTest 4.1.

\subsection{Experimental Scheme and Hypothesis}

(1) Take the different parts of the upper, middle and lower parts of the complete tobacco leaf to carry out the fracture tensile test respectively, see the cut part of the tobacco slice as shown in figure 1 below.

(2) The veins are analyzed separately from primary veins and secondary veins, because of the thickness of the petiole part and the tail part of the main vein, there are obvious differences after measurement, the diameter of the thickest end is about $5 \mathrm{~mm}$, and the thinest end is about $1 \mathrm{~mm}$. The length of the entire tobacco leaf has been measured above, however, the entire main leaf vein almost penetrates the entire tobacco leaf, and the diameter of the petiole and leaf tail drops by one millimeter every $90 \mathrm{~mm} \sim 110 \mathrm{~mm}$. Therefore, the main leaf vein is divided into 5 segments ( $\mathrm{A} \sim \mathrm{E}$ is broken, the diameter 
decreases in order), and the tensile test is carried out separately. See the sectioned cutout part of the tobacco stem as shown in figure 2 below.

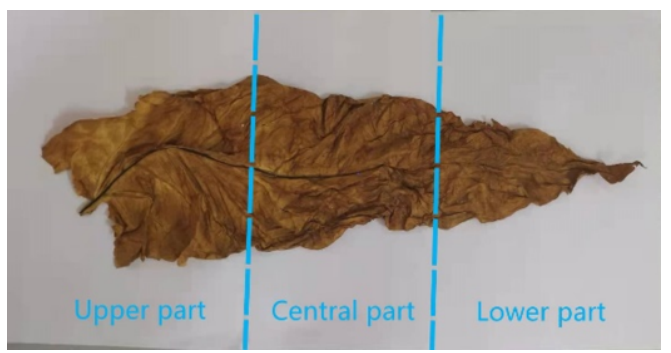

Figure 1. Location of cigarette slice interception.

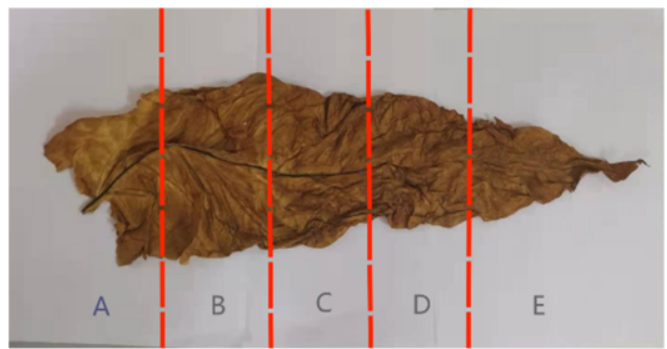

Figure 2. Simplified model diagram of tobacco leaf.

Four experimental hypotheses are proposed before the mechanical fracture experiment of tobacco slices and tobacco stems:

1) It is assumed that the material properties of the tobacco stems on the same tobacco leaf are the same, and the material properties of the tobacco slices on different parts of the same tobacco leaf are the same.

2) It is assumed that the tobacco leaves and stems are clamped sufficiently tightly with the tensile chuck, and the friction between the chuck and the chuck is negligible.

3) Because the thickness of the tobacco leaf in different parts of the same tobacco leaf is different, its thickness is the thickness of the upper top leaf $>$ the thickness of the middle waist $>$ the thickness of the lower foot leaf. It is assumed that the tobacco leaf fragments cut at different parts are uniform and the thickness is equal;

4) It is assumed that the density of each part of the same tobacco leaf is uniform.

\subsection{Solving the Parameters of Mechanical Index}

The electronic universal tester can apply tension to the tobacco leaf material or the tobacco stem material through the chuck, so as to realize the tensile fracture experiment of the material. Through the connection of the computer, the tensile amount and the tensile force data in the experiment can be recorded, and according to the formula:

$$
\left\{\begin{array}{l}
\sigma=\mathrm{F} / \mathrm{A} \\
\varepsilon=\Delta \mathrm{L} / \mathrm{L}
\end{array}\right.
$$


The stress and strain values of the material can be obtained. Where $\mathrm{F}$ is the force of the material, $\mathrm{A}$ is the cross-sectional area of the material, $\Delta \mathrm{L}$ is the elongation of the material, $\mathrm{L}$ is the original length of the material, $\sigma$ is the stress of the material, $\varepsilon$ is the strain of the material, and Young's modulus of the material:

$$
\mathrm{E}=\frac{\sigma}{\varepsilon}=\frac{\mathrm{F} / \mathrm{A}}{\Delta \mathrm{L} / \mathrm{L}}=\frac{\mathrm{F} \cdot \mathrm{L}}{\mathrm{A} \cdot \Delta \mathrm{L}}
$$

For the solution of the tensile strength, through the tensile fracture experiment, the force curve with time is obtained. Fmax is the value of the force corresponding to the highest point in the curve ,The cross-sectional area of the tobacco stem fracture section is $\mathrm{A}$, and the formula for calculating the tensile strength:

$$
\sigma=\frac{F_{\max }}{A}
$$

For the solution of the elongation $\delta$, elongation $\delta$ refers to the index describing the plasticity of a material. Through tensile fracture experiment, the percentage ratio between the total deformation $\Delta \mathrm{L}$ and the original distance $\mathrm{L}$ of tobacco stem after tensile fracture is as follows:

$$
\delta=\frac{\Delta L}{L} \times 100 \%
$$

In the experiment of measuring the mechanical properties of tobacco, it mainly relies on the records of Material Test software in the computer. The tension-displacement data of blade and stem were obtained by electronic universal experiment machine, since the range of the selected stretching equipment can be adjusted, the ranges of the equipment are $50 \mathrm{~N}, 100,200 \mathrm{~N}, 500 \mathrm{~N}, 1 \mathrm{KN}$, the measurement range of large deformation is $10 \sim 600 \mathrm{~mm}$, the resolution of large deformation measurement is $0.008 \mathrm{~mm}$, and the force accuracy is in Within the range of $0.001 \mathrm{~N}$, Therefore, the measurement results of the equipment are relatively accurate. The equipment parameter setting of the $50 \mathrm{~N}$ range is selected when the tobacco sheet is stretched, and the equipment parameter setting of the $200 \mathrm{~N}$ range is selected when the tobacco stem is stretched and broken. Because of the geometrical characteristics of the experimental samples have no effect on the result value, according to the above experimental plan, when measuring the data, the parts of the tobacco leaves without stalks are cut into rectangular pieces with a side length of $50 \mathrm{~mm} \times 80 \mathrm{~mm}$, and the leaf stems are cut into long pure stem with a length of $100 \mathrm{~mm}$, use clips to fix the short sides on both ends of the chuck of the electronic universal testing machine to make it slack, as shown in figure 3 below. 


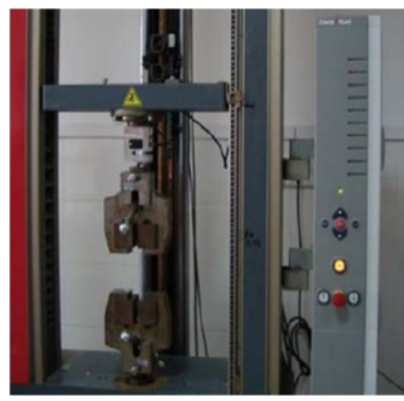

(a) Stretching equipment

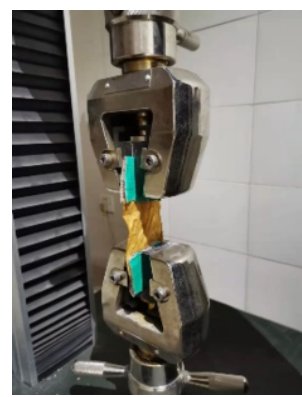

(b) Tobacco clamping

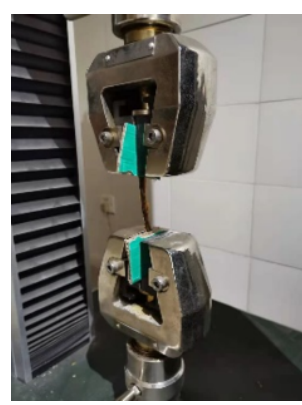

(c) Tobacco stem clamping

Figure 3. Tobacco leaf and tobacco stem clamping method.

\subsection{Experimental Data}

The data directly derived from the experimental equipment include breaking force, maximum force, tensile strength, tensile yield stress (the ratio of the maximum force to the area of the fractured section), tensile yield strain (the displacement corresponding to the maximum tensile force is compared with the original Length ratio), maximum displacement (used to calculate extension Elo2ngation) and tensile fracture stress (ratio of fracture force to fracture cross-sectional area). According to these indirect or directly derived parameters that reflect the mechanical properties of tobacco leaves, the 5 groups (a total of 30 tobacco leaves) experimental data are plotted in the table 1 below. In each group of data, The values tobacco stem segments and tobacco sheet are all averaged. This table reflects the mechanical properties of tobacco leaves and stems with a moisture content of $18 \%$ and a temperature of $50^{\circ} \mathrm{C}$. See table 1 below.

Table 1. Parameters of tobacco leaf and tobacco stalk.

\begin{tabular}{|c|c|c|c|c|c|c|c|c|c|}
\hline \multicolumn{2}{|c|}{ group } & $\begin{array}{l}\text { parame } \\
\text { ter }\end{array}$ & $\begin{array}{l}\text { Fractu } \\
\text { re } \\
\text { force/ } \\
\mathrm{N}\end{array}$ & $\begin{array}{c}\text { Fracture } \\
\text { stress/M } \\
\text { pa }\end{array}$ & $\begin{array}{l}\text { Maximu } \\
\mathrm{m} \text { force } \\
\text { /N }\end{array}$ & $\begin{array}{c}\text { Tensile } \\
\text { strength/M } \\
\text { pa }\end{array}$ & $\begin{array}{c}\text { Maximum } \\
\text { displacement/ } \\
\mathrm{mm}\end{array}$ & $\begin{array}{l}\text { Yiel } \\
\text { d } \\
\text { stra } \\
\text { in }\end{array}$ & $\begin{array}{c}\text { Elong } \\
\text { ation } \\
\%\end{array}$ \\
\hline \multirow{6}{*}{$\begin{array}{l}\text { Gro } \\
\text { up } 1\end{array}$} & \multirow{5}{*}{$\begin{array}{c}\text { Tobacc } \\
\text { o stem } \\
\text { segmen } \\
\text { ts }\end{array}$} & A & 44.23 & 2.25 & 128 & 6.75 & 4.28 & 0.04 & 0.04 \\
\hline & & B & 26.36 & 2.09 & 98 & 7.79 & 3.89 & 0.04 & 0.04 \\
\hline & & $\mathrm{C}$ & 14.24 & 1.99 & 75 & 10.61 & 3.91 & 115 & 0.04 \\
\hline & & $\mathrm{D}$ & 7.36 & 2.34 & 49 & 15.50 & 3.59 & 0.03 & 0.03 \\
\hline & & $\mathrm{E}$ & 1.80 & 2.30 & 18 & 23 & 4.35 & 0.04 & 0.04 \\
\hline & $\begin{array}{c}\text { Tobacc } \\
\text { o } \\
\text { sheet }\end{array}$ & $\begin{array}{l}\text { avera } \\
\text { ge } \\
\text { value }\end{array}$ & 0.67 & 0.15 & 2.57 & 0.59 & 5.15 & 0.06 & 0.06 \\
\hline \multirow{6}{*}{$\begin{array}{l}\text { Gro } \\
\text { up } 2\end{array}$} & \multirow{5}{*}{$\begin{array}{c}\text { Tobacc } \\
\text { o stem } \\
\text { segme } \\
\text { nts }\end{array}$} & $\mathrm{A}$ & 42.32 & 2.15 & 129.50 & 6.60 & 4.32 & 0.03 & 0.04 \\
\hline & & B & 25.23 & 2.01 & 99.10 & 7.88 & 3.92 & 0.03 & 0.04 \\
\hline & & $\mathrm{C}$ & 13.63 & 1.92 & 75.90 & 10.70 & 3.94 & 0.03 & 0.04 \\
\hline & & D & 7.05 & 2.26 & 49.50 & 15.70 & 3.62 & 0.03 & 0.04 \\
\hline & & $\mathrm{E}$ & 1.72 & 2.13 & 18.20 & 23.05 & 4.40 & 0.03 & 0.04 \\
\hline & $\begin{array}{c}\text { Tobacc } \\
\text { o } \\
\text { sheet }\end{array}$ & $\begin{array}{c}\text { avera } \\
\text { ge } \\
\text { value }\end{array}$ & 0.64 & 0.15 & 2.60 & 0.58 & 5.19 & 0.06 & 0.06 \\
\hline
\end{tabular}




\begin{tabular}{cccccccccc}
\hline & & A & 38.35 & 10.37 & 127.48 & 15.36 & 4.33 & 0.03 & 0.04 \\
Gro & Tobacc & B & 22.85 & 90 & 97.60 & 127 & 3.94 & 0.03 & 0.04 \\
up 3 & C stem & C & 12.35 & 85 & 74.71 & 120 & 3.96 & 0.04 & 0.04 \\
& $\begin{array}{c}\text { segme } \\
\text { nts }\end{array}$ & D & 6.39 & 1.16 & 48.80 & 1.17 & 3.73 & 0.03 & 0.04 \\
& E & 1.57 & 10.37 & 17.98 & 15.36 & 4.23 & 0.03 & 0.04 \\
\cline { 2 - 9 } & $\begin{array}{c}\text { Tobacc } \\
\text { o } \\
\text { sheet }\end{array}$ & $\begin{array}{c}\text { avera } \\
\text { ge } \\
\text { value }\end{array}$ & 0.58 & 0.16 & 2.55 & 0.57 & 5.25 & 0.06 & 0.07 \\
\hline
\end{tabular}

Table 1. Continue

\begin{tabular}{|c|c|c|c|c|c|c|c|c|c|}
\hline \multirow{6}{*}{ Group 4} & \multirow{5}{*}{$\begin{array}{l}\text { Tobacco } \\
\text { stem } \\
\text { segments }\end{array}$} & A & 52.19 & 10.37 & 110.33 & 15.358 & 4.39 & 0.04 & 0.04 \\
\hline & & B & 31.11 & 90 & 84.47 & 127 & 3.99 & 0.03 & 0.04 \\
\hline & & $\mathrm{C}$ & 16.80 & 85 & 64.65 & 120 & 4.02 & 0.03 & 0.04 \\
\hline & & D & 8.65 & 10.37 & 42.28 & 15.36 & 3.68 & 0.03 & 0.04 \\
\hline & & E & 3.12 & 1.15 & 15.56 & 1.17 & 4.46 & 0.04 & 0.04 \\
\hline & $\begin{array}{c}\text { Tobacco } \\
\text { sheet }\end{array}$ & $\begin{array}{c}\text { average } \\
\text { value }\end{array}$ & 0.79 & 0.17 & 2.23 & 0.50 & 5.21 & 0.06 & 0.07 \\
\hline \multirow{6}{*}{ Group 5} & \multirow{5}{*}{$\begin{array}{l}\text { Tobacco } \\
\text { stem } \\
\text { segments }\end{array}$} & $\mathrm{A}$ & 48.29 & 48.29 & 135.96 & 7.53 & 4.36 & 0.04 & 0.04 \\
\hline & & B & 28.78 & 28.78 & 104.07 & 106.4 & 4.01 & 0.03 & 0.04 \\
\hline & & C & 15.55 & 15.55 & 79.65 & 102.2 & 3.95 & 0.03 & 0.04 \\
\hline & & D & 8.04 & 8.04 & 52.38 & 1.60 & 3.62 & 0.03 & 0.04 \\
\hline & & $\mathrm{E}$ & 1.96 & 1.96 & 19.16 & 7.53 & 4.4 & 0.04 & 0.04 \\
\hline & $\begin{array}{c}\text { Tobacco } \\
\text { sheet }\end{array}$ & $\begin{array}{c}\text { average } \\
\text { value }\end{array}$ & 0.73 & 0.18 & 2.59 & 0.58 & 5.22 & 0.06 & 0.07 \\
\hline
\end{tabular}

Because of the part of the tobacco leaf can withstand a very small force when it is broken, it usually breaks at $2 \sim 3 \mathrm{~N}$. When measuring it with the electronic universal tester, due to the vibration of the machine itself, there will be a certain error in the idling situation. The vibration of the machine during operation will cause a white noise error value of $0.068 \sim 0.204 \mathrm{~N}$, see figure 4 below the mechanical vibration force diagram of the electronic universal testing machine at no load is shown.

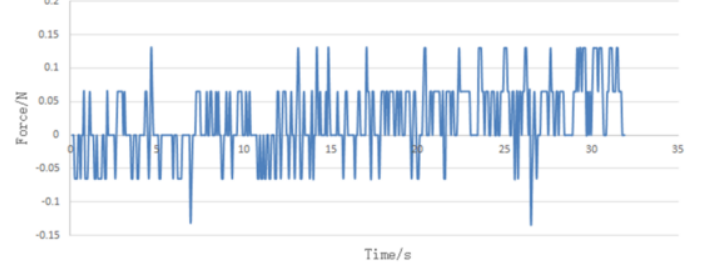

Figure 4. Vibration noise of the experimental instrument.

This error value will have a greater impact on the mechanical curve of the tobacco leaf, causing the force-displacement curve of the tobacco leaf segment to appear jagged, as shown in the tobacco segment diagram in figure 5 below. For the tobacco stem part, since the minimum force of the tobacco stem fracture is greater than $15 \mathrm{~N}$, the maximum force of fracture is in the range of $95 \sim 130 \mathrm{~N}$ (different diameters have different maximum fracture forces), the error caused by the noise and vibration of the machine can be ignored. Take one group of tobacco stem tensile fracture force curves with time, as shown in 
figure 5 below, a, b, c, d, e correspond to each part of the tobacco stem, and the diameter decreases in order.

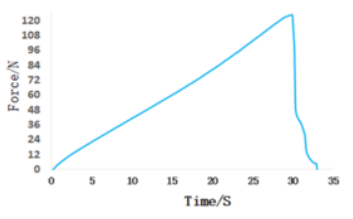

(Tobacco stem a)

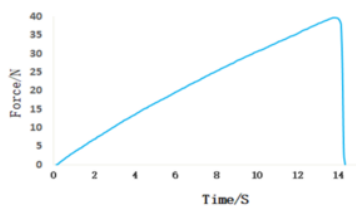

(Tobacco stem d)

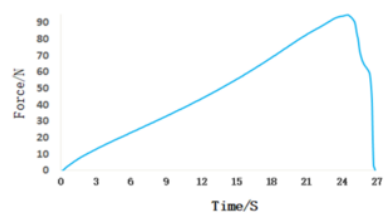

(Tobacco stem b)

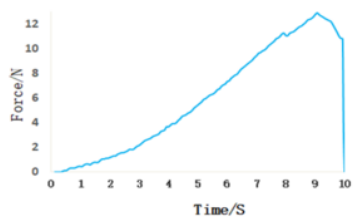

(Tobacco stem e)

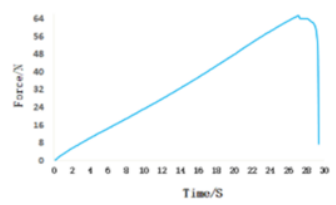

(Tobacco stem c)

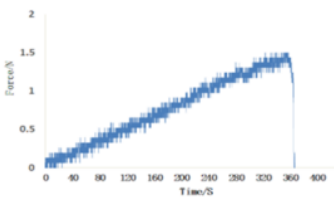

(Tobacco stem $\mathrm{f}$ )

Figure 5. Variation of tobacco stem and fragment force with displacement in each segment.

When the upper puller of the tensile testing machine is tensile loaded at a fixed speed, the tensile force-displacement curve of the main vein derived from the data is shown in figure 5. It can be seen that the tensile force-displacement curve can be divided into 4 Stages. In the first stage (equipment start-up stretch -> elastic modulus calibration starting point), the sample is lifted under the action of the upper slider, the sample slips in the clamp a little, and the sample is straightened. The second stage (start point of elastic modulus calibration -> end point of elastic modulus calibration), in this stage, the sample is in the elastic stage, the tensile force and deformation are approximately linearly changed. The end point of elastic modulus calibration corresponds to Is the maximum yield load. In the third stage (the end point of elastic modulus calibration $->$ the maximum force point), the tensile force increases with the deformation, and the tissue in the main vein begins to fracture, this stage is the non-linear failure stage, at the highest point of the curve (that is, the maximum force point) The sample fracture and the material fails. In the fourth stage (the point of maximum force $->$ the tensile force drops to 0 ), the sample breaks and the load is unloaded.

The data derived from the experiment, through the calculation and measurement of tobacco stalk and tobacco simulation required parameters range. Shown in table 2 below. Through sampling measurement and data calculation of the same batch of heated and humidified tobacco leaves, finally obtained parameter data reflecting the mechanical properties at a temperature of $50^{\circ} \mathrm{C}$ and a moisture content of $18 \%$. Calculate the tobacco leaves and stems at equal intervals and remove the values with large deviations in heating and humidifying equipment. The numerical range of various parameters in tobacco leaf and tobacco stem was obtained, as shown in table 2. 
Table 2. Material attribute parameters of tobacco leaf.

\begin{tabular}{ccc}
\hline (Materials) & ( Parameters) & (Value Range) \\
\hline \multirow{2}{*}{ Tobacco leaf material parameters } & Yield strain & $0.06 \sim 0.07$ \\
& Maximum force $/ \mathrm{N}$ & $1.90 \sim .2 .80$ \\
& Fracture stress $/ \mathrm{MPa}$ & $0.53 \sim 0.86$ \\
& Young's modulus $/ \mathrm{MPa}$ & $10 \sim 15$ \\
& Tensile strength / MPa & $0.53 \sim 0.93$ \\
& Density /(kg·m ${ }^{3}$ ) & $300 \sim 380$ \\
& Fracture force $/ \mathrm{N}$ & $0.85 \sim 1.35$ \\
\hline Material parameters of tobacco & Fracture force $/ \mathrm{N}$ & $34.29 \sim 42.21$ \\
stem & Maximum force $/ \mathrm{N}$ & $120.85 \sim 130.93$ \\
& Fracture stress $/ \mathrm{MPa}$ & $2.32 \sim 4.38$ \\
& Young's modulus $/ \mathrm{MPa}$ & $130 \sim 150$ \\
& Tensile strength / MPa & $6.52 \sim 22.65$ \\
& Density / $/ \mathrm{kg} \cdot \mathrm{m}^{3}$ ) & $636 \sim 932$ \\
& Yield strain & $0.03 \sim 0.06$ \\
\hline
\end{tabular}

\section{Simulation of Tobacco Leaves Tensile Fracture}

\subsection{Fracture Simulation Analysis of Tobacco Leaves}

In the process of studying the mechanical properties of tobacco leaves, the process of tensile fracture and shear fracture of tobacco leaves is involved. However, one of the commonly used methods to solve the fracture problem in the extended finite element method is to adapt to the mesh without re-meshing. The lattice geometry is discontinuous, so it is mainly used for discretization expansion. Therefore, this paper chooses the XFEM method for calculation and analysis.

The function used by the extended finite element method is the nodal extension function. In order to realize the fracture analysis, the extension function usually includes the crack tip asymptotic function and the discontinuous function. The displacement vector function using the overall division characteristic can be expressed as $\mathrm{u}$ as [13]:

$$
\mu=\sum_{i=1}^{N} N_{i}(x)\left[\mu_{i}+H(x) a_{i}+\sum_{\alpha=1}^{4} F_{\alpha}(x) b_{i}^{\alpha}\right]
$$

$N_{i}(x)$ Is the finite element node displacement shape function; $u_{i}$ is the finite element displacement vector to solve the corresponding continuous part; $a_{i}$ is the node expansion degree of freedom vector related to the crack surface; $H(x)$ is the discontinuous jump function along the crack surface; $b_{i}^{\alpha}$ is the node related to the crack The extended degree of freedom vector; $F_{\alpha}(x)$ is the asymptotic function near the crack.

The model is meshed before the crack propagation. There are two forms of crack propagation: one is to expand along the element and the other is the collection of crack lines through a certain element [14-15], as shown in figure 6 . In formula (5), $u_{i}$ it is 
applicable to all nodes in the model, $\mathrm{H}(\mathrm{x}) a_{i}$ only the nodes whose shape function is passed through the element by the crack surface, and the nodes whose shape function is in the crack tip element.

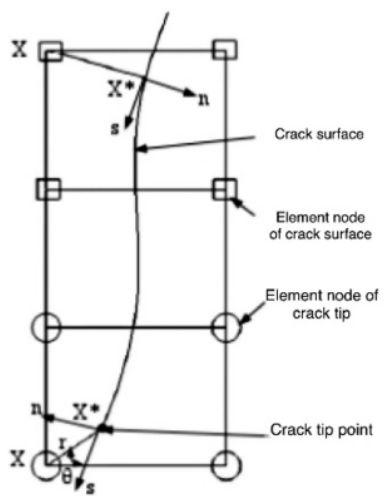

Figure 6. Schematic diagram of the crack surface passing through the element.

The discontinuous jump function $\mathrm{H}(\mathrm{x})$ along the crack surface, $\mathrm{H}(\mathrm{x})$ above the crack is set to 1 , and $\mathrm{H}(\mathrm{x})$ below the crack is set to -1 , which can be expressed by the following formula:

$$
H(x)=\left\{\begin{array}{cc}
1 & \left(X-X^{*}\right) n \geq 0 \\
-1 & \text { otherwise }
\end{array}\right.
$$

Where: $\mathrm{X}$ is the sample point; $X^{*}$ is the point closest to $\mathrm{x}$ on the crack surface; $\mathrm{n}$ is the unit normal vector at the point $X^{*}$. The crack tip asymptotic function $F_{\alpha}(x)$ can improve the calculation accuracy. The crack tip asymptotic function $F_{\alpha}(x)$ can be expressed as:

$$
F_{i}^{\alpha}=\sqrt{r}\left[\sin \frac{\theta}{2}, \cos \frac{\theta}{2}, \sin \theta \sin \frac{\theta}{2}, \sin \theta \cos \frac{\theta}{2}\right]
$$

Where: $(r, \theta)$ is the polar coordinate system; $\theta$ is the angle between the line segment composed of the element node where the crack tip is located and the crack tip point and the unit tangential vector at the crack tip point.

When material properties of the tobacco leaves are known, replace the tobacco leaves with a thin slice of the same size and thickness in the experimental part to conduct tensile fracture simulation. When crack growth analysis is performed by the finite element method, components need to be pre-cracked (figure 7) The drawing and tearing process of the smoke sheet is obtained by post-simulation processing and the unit in the figure is Mpa.

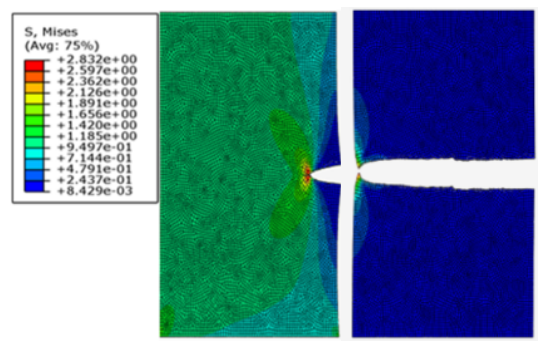

Figure 7. Tensile fracture process diagram of the tobacco leaves. 
The force-time curve and stress-strain curve of an element above the crack of tobacco leaves are shown in figure 8 and 9.

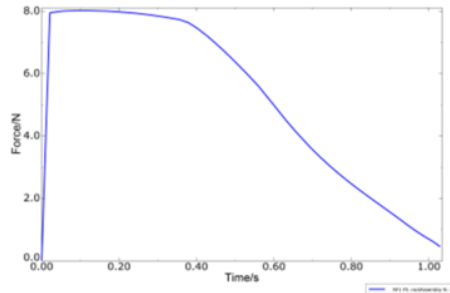

Figure 8. Force-time diagram.

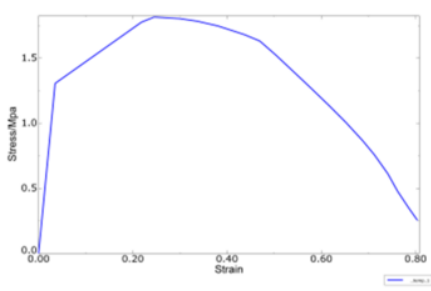

Figure 9. Stress-strain curve.

\subsubsection{Stress Intensity Factor at the Tip of the Tobacco Leaves Crack.}

Fracture mechanics has been proposed since the 1950s, and developed into a real discipline in just a few decades. In 1921, Griffith[16] proposed the energy criterion for crack growth of brittle materials, condition of crack propagation is that the crack propagation force is more than the propagation resistance. In 1948, Orowan[17] and Irwin[18] studied the crack propagation of plastic materials, modified the energy theory formula proposed by Griffith. In 1957, Irwin[19] put forward the viewpoint of the stress field intensity of the elastic body crack tip attachment, and established a fracture criterion based on the stress intensity factor[20]. In theoretical analysis of fracture mechanics, Wright and Vincent[21] reviewed the fracture mechanism of biological materials, and mentioned that biological materials have three crack propagation methods:(I) stretching;(II) in-plane shear(edge slip);(III) Out-of-plane shear(tear). As soon as the fracture be-gins, the crack will spread in one of the ways. The following will simulate three types of fracture methods through tobacco leaf fragments. In most cases, it is still a mixed form of type I and type II cracks. As long as the stress field components of type I and type II crack are superimposed, the stress field component can be obtained as formula(8) and the displacement field component near the crack tip as formula(9). Among them, the tensile and tearing process of the sheet is the generation and propagation of cracks, which are compound cracks[22].

For the penetrating crack with a length of $2 \mathrm{a}$ in infinite plate shown in figure 10 , when the distance is subjected to a bidirectional uniform tensile stress $\sigma$, the displacement field and stress field components near crack tip are as follows.

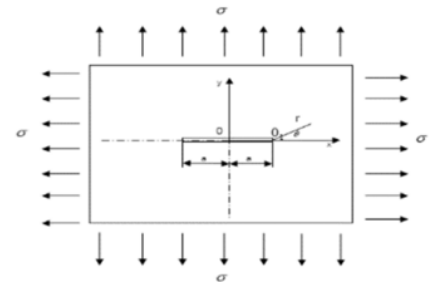

(a) Type I crack

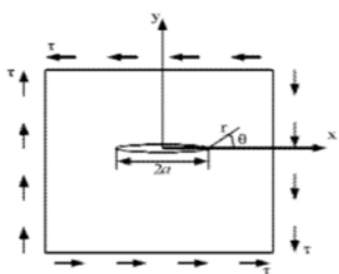

(b) Type II crack

Figure 10. Mixed type crack.

$$
\begin{gathered}
\sigma_{x x}(r, \theta)=\frac{K_{I}}{\sqrt{2 \pi r}} \cos \frac{\theta}{2}\left(1-\sin \frac{\theta}{2} \sin \frac{3 \theta}{2}\right)-\frac{K_{I I}}{\sqrt{2 \pi r}} \sin \frac{\theta}{2}\left(2+\cos \frac{\theta}{2} \cos \frac{3 \theta}{2}\right) \\
\sigma_{y y}(r, \theta)=\frac{K_{I}}{\sqrt{2 \pi r}} \cos \frac{\theta}{2}\left(1+\sin \frac{\theta}{2} \sin \frac{3 \theta}{2}\right)+\frac{K_{I I}}{\sqrt{2 \pi r}} \sin \frac{\theta}{2} \cos \frac{\theta}{2} \cos \frac{3 \theta}{2}
\end{gathered}
$$




$$
\begin{gathered}
\sigma_{x y}(r, \theta)=\frac{K_{I}}{\sqrt{2 \pi r}} \cos \frac{\theta}{2} \sin \frac{\theta}{2} \sin \frac{3 \theta}{2}+\frac{K_{I I}}{\sqrt{2 \pi r}} \cos \frac{\theta}{2}\left(1-\sin \frac{\theta}{2} \sin \frac{3 \theta}{2}\right) \\
u_{x}=\frac{K_{I}}{4 \mu} \sqrt{\frac{r}{2 \pi}}\left[(2 k-1) \cos \frac{\theta}{2}-\cos \frac{3 \theta}{2}\right]+\frac{K_{I I}}{4 \mu} \sqrt{\frac{r}{2 \pi}}\left[(2 k+3) \sin \frac{\theta}{2}+\sin \frac{3 \theta}{2}\right] \\
u_{y}=\frac{K_{I}}{4 \mu} \sqrt{\frac{r}{2 \pi}}\left[(2 k+1) \sin \frac{\theta}{2}-\sin \frac{3 \theta}{2}\right]-\frac{K_{I I}}{4 \mu} \sqrt{\frac{r}{2 \pi}}\left[(2 k-3) \cos \frac{\theta}{2}+\cos \frac{3 \theta}{2}\right]
\end{gathered}
$$

In the formula $k=3-4 v / 1+v$ (Plane stress situation); $k=3-4 v$ (Plane strain situation); $\mathrm{G}$ represents the shear modulus of the material, $G=E / 2(1+v)$; The stress intensity factor $\mathrm{K}$ is defined according to the following formula (10) :

$$
\begin{gathered}
K_{I}=\lim _{r \rightarrow 0} \sqrt{2 \pi r \sigma_{y}} \mid \theta=0 \\
K_{I I}=\lim _{r \rightarrow 0} \sqrt{2 \pi r \tau_{x y}} \mid \theta=0
\end{gathered}
$$

For the crack problem shown in figure 10, the stress intensity factor is generally determined by: the shape, size and direction of the crack; the magnitude and direction of the applied load; Sometimes it is also related to certain constants of the material (in the problem involving displacement boundary conditions). Its dimension is $M p a \sqrt{m}$.

\subsubsection{The Influence of Different Crack Angles to the Stress Intensity Factor.}

The previous section described the solving method and theoretical knowledge for the stress field and stress intensity factor of the compound plane crack tip. In order to analyze deeply the tip stress intensity factor K of crack tip in the tobacco leaf fragment, the central oblique crack should be selected. When the angle between the oblique crack and the $\mathrm{X}$ axis, that is the crack inclination angle takes different values(when $\theta$ equal to $0^{\circ} 15^{\circ} 30^{\circ} 45^{\circ} 60^{\circ} 75^{\circ} 90^{\circ}$ ), The XFEM method is used to calculate the stress intensity factor. When solving the stress intensity factor, it is only necessary to set the simulation to not allow the expansion of cracks. The mesh size of the entire area is about $0.2 \mathrm{~mm}$, and the entire model has 37500 elements, The unit type is three-dimensional 8-node bricks (C3D8R). Analysis method adopts static elastic analysis. After pre-cracking the stalked to-bacco segments at different angles, under the same grid size and the same applied load, the cracks did not expand after the end of the simulation, as shown in figure 11 below.

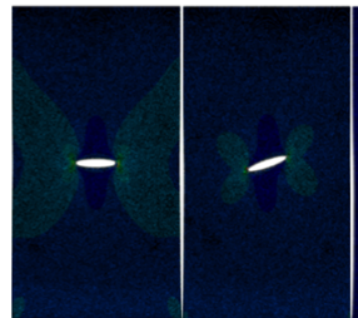

$\left(0^{\circ}\right)$ $\left(15^{\circ}\right)$

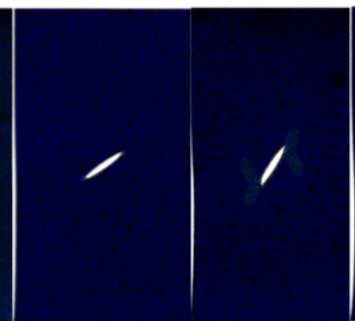

$\left(30^{\circ}\right)$ $\left(45^{\circ}\right)$

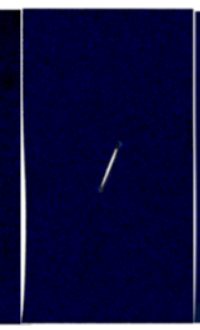

$\left(60^{\circ}\right)$

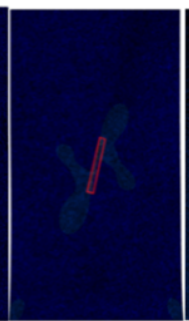

$\left(75^{\circ}\right)$

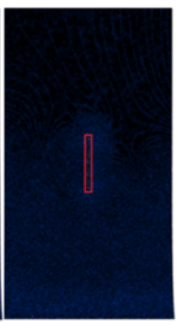

$\left(90^{\circ}\right)$

Figure 11. Analysis diagram of forces from different angles.

After opening the generated dat file with Notepad, you can see the fracture stress intensity factor. Take the size of the stress intensity factor generated by the last step, the difference of the corresponding stress intensity factor $\mathrm{K}$ and the size for comparison. The 
data table is shown in table 3 below.

Table 3. Influence of crack Angle on stress intensity factor.

\begin{tabular}{lllccccc}
\hline$\theta$ & $0^{\circ}$ & $15^{\circ}$ & $30^{\circ}$ & $45^{\circ}$ & $60^{\circ}$ & $75^{\circ}$ & $90^{\circ}$ \\
\hline$K_{I}$ & 2.606 & 2.0378 & 1.3955 & 0.9096 & 0.5265 & 0.3309 & 0 \\
$K_{\mathrm{II}}$ & 0 & 0.2892 & 0.4736 & 0.6185 & 0.4807 & 0.3278 & 0 \\
\hline
\end{tabular}

\subsection{Fracture and Tensile Simulation of Tobacco Stem}

By a stroke of tensile experiment of peduncle constitutive stress strain curve, in the elastic stage and plastic stage, stage of damage of the stress value with an approximate numerical range, below the experimental simulation of the stress-strain curve, due to the smaller peduncle, its shape is similar to cylindrical, maximum diameter of $5 \mathrm{~mm}$ or so, The smallest end is about $1 \mathrm{~mm}$, so in the finite element simulation, the shape of the tobacco stem is like a cylinder. The grid size of the whole area is about $0.2 \mathrm{~mm}$, and the unit type is three-dimensional8-Nodebricks (C3D8R). The simulation results are analyzed, and the force-time variation diagram and stress-strain curve are derived. According to the stress-strain curve obtained, the stress-strain curve is compared with the stress-strain curve obtained from the experiment. In terms of material property setting, In addition to the elastic setting, there are also plastic Material (Yieldstress, PlasticStrain) as well as the DuctileDamage (FRuctiledamage, Stresstria in DamageForeducation aterialXIality, StrainRate), among which YieldStress, PlasticStrain, FRINGEALITRIE, Stress triaxiality and StrainRate can be read directly according to the above stress-strain diagram. Finally, the read data are filled into the material property Settings required for the fracture tensile simulation of tobacco stem below. After the simulation is completed, figure 12 is the tensile deformation diagram of each section and the unit in the figure is Mpa. A unit at the deformation location is selected to draw the curve of output and time (figure 13) and the curve of stress and strain (figure 14) through the process output.

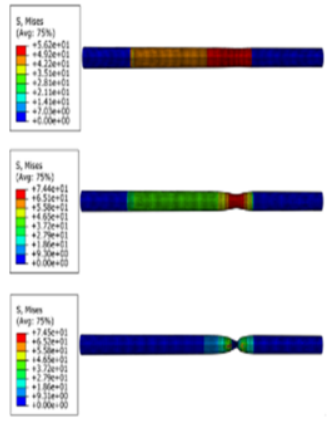

(a segment of tobacco stem)

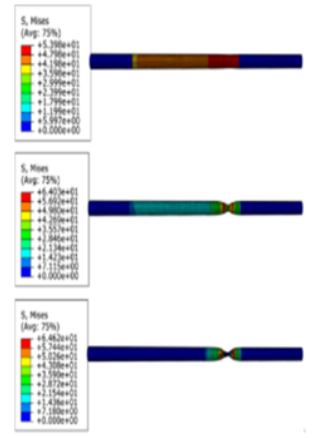

(b segment of tobacco stem)

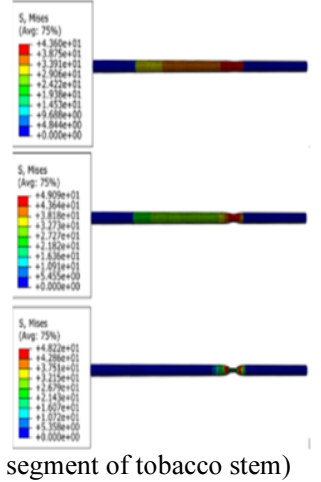



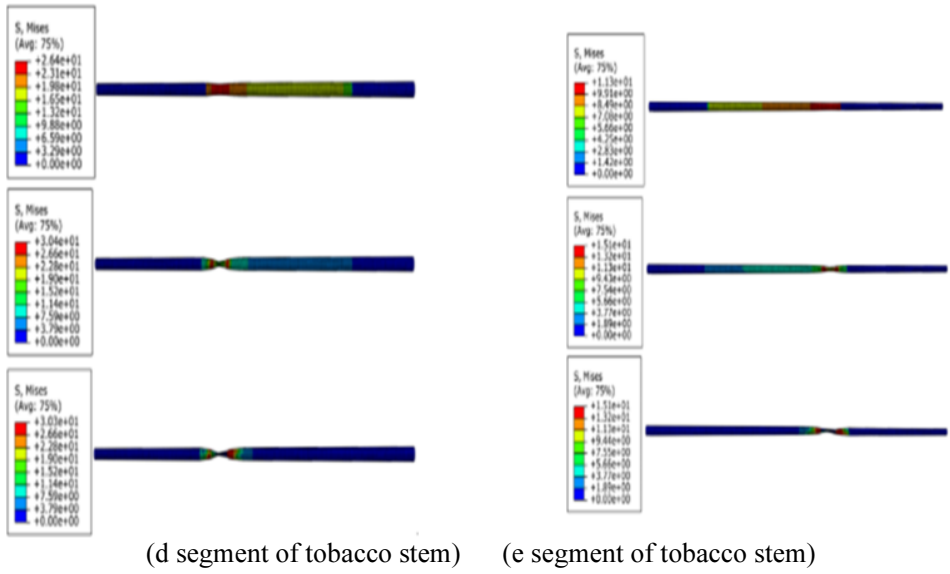

Figure 12. Tensile deformation diagram of tobacco stem.

Figure 12 shows the tensile deformation process of tobacco stem in sections A, B, $\mathrm{C}, \mathrm{D}$ and $\mathrm{E}$ respectively. Because the diameters of both ends are different, the tensile deformation will occur at the thinner end, and the simulation deformation process is consistent with the fact. It can be seen from the figure that as the analysis steps increase or the time increases, the deformation of the tobacco stem becomes larger and larger, and the greater the deformation, the greater the tensile force.

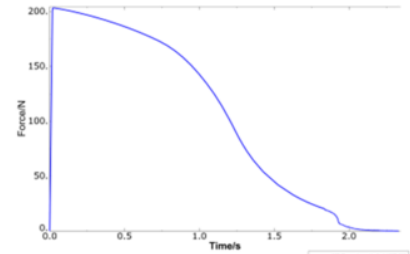

(segment a)

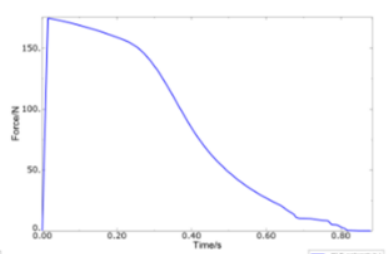

(segment b)

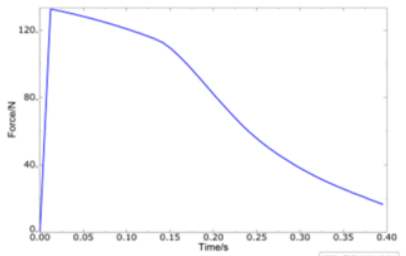

(segment c)

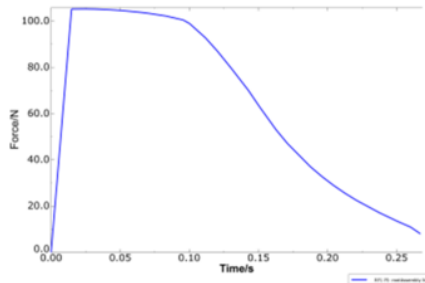

(segment d)

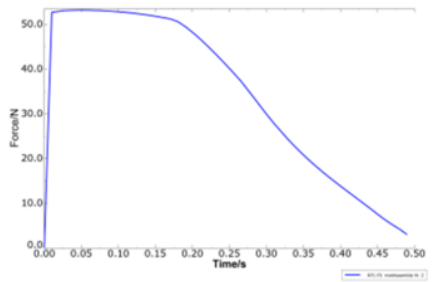

(segment e)

Figure 13. Tobacco stem force - time change diagram.

Figure 13 paragraphs force variation over time, from figure 13 paragraphs peduncle force changing with time curve, the tensile deformation process can be classified into three stages, and the experiment of force variation with time a total of four stages, is so peduncle experiments of tensile process is divided into four stages, because the beginning of the first stage peduncle tensile process, peduncle sample, Peduncle a little slip inside the holder, peduncle was straightening process for the first stage, here was caused by external factors, and the simulation is not these errors, peduncle tensile deformation process experienced three stages and peduncle laboratory tensile fracture after three stages of the process has the same status, from elastic stage to stage of plastic deformation and damage failure stage. 
Difference: there is a deviation between the experiment and the simulation, the experimental damage stage is more obvious, the failure fracture time is a little longer, and the tobacco stem in the tensile experiment after the molding stage will break instantly, the simulation is relative to the reality partial beautification. In addition, the force variation over time obtained by simulation post-processing shows that the maximum force of each section of tobacco stem is larger than the maximum force obtained from the test, which is probably more than $20 \sim 30 \mathrm{~N}$, which is an inevitable error.

By comparing a stroke unit force changing with time curve and the smoke breaks section obtained above force variation over time (figure 8), through the force variation with time, tobacco used by tensile strength is far less than peduncle fracture the use of force, it also indirectly verified the peduncle of crack propagation of the tobacco leaf block, And the crack propagation of tobacco leaf tends to propagate along the edge of tobacco stem.

By comparing the force-time variation diagram of tobacco stem tensile fracture obtained in the above experiment, it is also obvious that the minimum force required by tobacco stem fracture is far more than the maximum force required by tobacco stem fracture, which directly proves that tobacco stem has a certain retardation effect on tobacco leaf crack growth.

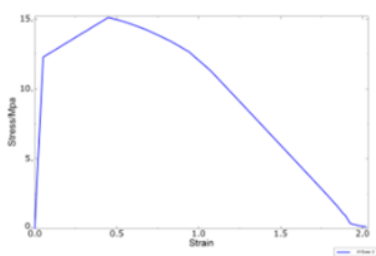

(a)

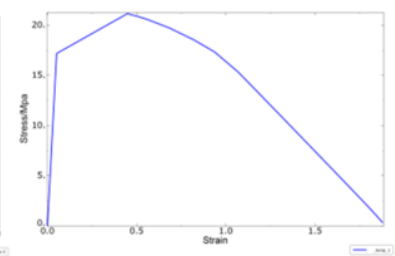

(b)

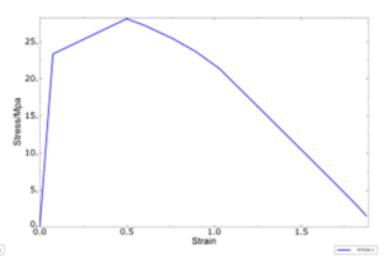

(c)

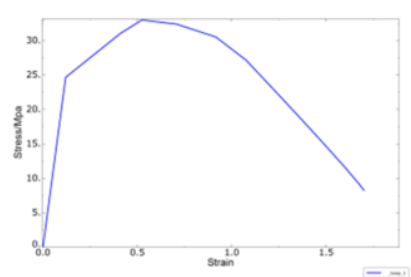

(d)

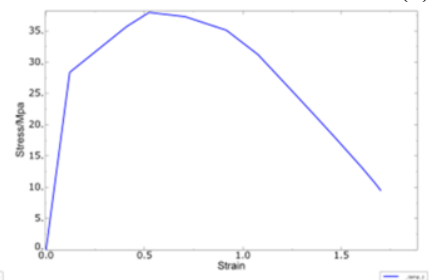

(e)

Figure 14. Stress-strain curve of tobacco stem.

Figure 14 shows the stress-strain variation curves of tobacco stem under tensile deformation in sections A, B, C, D and E respectively. As can be seen from the figure, with the increase of analysis steps or time, the larger the deformation is, the greater the tension will be, and the corresponding stress will gradually increase. When damage and fracture occur, the tension will begin to decrease, and the corresponding stress will also gradually decrease until the complete fracture, and the tension and stress will become 0 .

By analyzing the above stress-strain curve, the three stages of tobacco stem tensile fracture can be read out more obviously through the trend of the curve, and the change of constitutive relation in the tensile process of tobacco stem can be described more vividly by the tensile stress-strain curve. 


\subsection{Simulation Analysis of Fracture of Whole Tobacco Leaf}

The above section is the tensile simulation analysis of the tobacco leaf fragments and the tobacco stem part, through the combination analysis of the simulation results and the data derived from the experimental part. The force required to pull the tobacco stems is much greater than the force required to break the mesophyll after the stem removal, which indirectly verifies that the stems have a blocking effect on the crack propagation of the tobacco leaves. In the process of research on the fracture behavior of plant leaves, from the perspective of macro mechanics, the structure of the leaf adopts a smooth transitional assembly mode to connect with the whole package, and the longitudinal and transverse veins are interwoven and distributed, and the hard vascular bundles "leaf veins" And the soft "mesophyll" composition, the two can also be regarded as a certain rigid-flexible matching connection, which effectively reduces the stress concentration of the blade and the initiation of cracks. The principle of leaf crack arrest is similar to that of insect wings. Cracks will be blocked or deflected at the interface between leaf veins and mesophyll, which can effectively resist fatigue and cracking under the action of natural environment, as shown in figure 15 below. This section mainly uses the simulation of the I-type crack propagation to verify that the tobacco stems play a role in retarding the crack propagation when the tobacco leaves are torn by force. In order to facilitate the establishment of the model, without affecting the analysis results and the application of force. Since the thickness of the third-level veins is very close to the thickness of the leaf surface itself, the stalks of each level after the second-level can be ignored to facilitate the division of the mesh. The secondary tobacco stems are drawn only a little symbolically, and the tobacco leaf model is simplified without affecting the simulation results. The length and width of the tobacco leaf are derived from physical measurements of the tobacco leaf. After modeling, import the exported igs file into the finite element software ABAQUS, and then set the material properties, set the pre-crack, mesh division, boundary conditions and load application on the model, as shown in the following figure 16 mesh and blade force diagram.

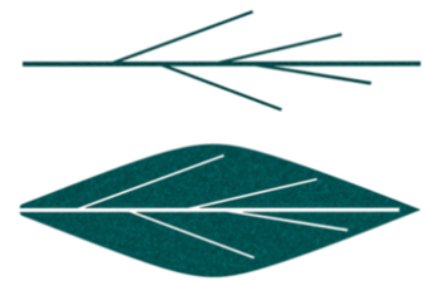

Figure 15. Arrest behavior of tobacco veins.

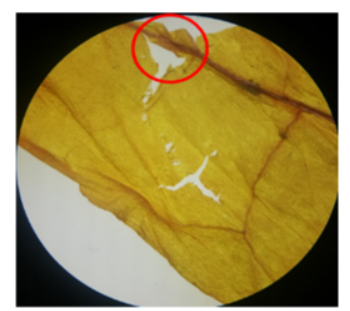

Figure 16. Grid and force diagram.

After the pre-processing work is completed and submitted, the simulation results are obtained after post-processing calculations. After the pre-processing part of the finite element analysis is set up, enter the post-processing module, and submit the calculation after entering the job. The total calculation time is $1 \mathrm{~s}$. After the calculation is completed, it is obtained that under the pre-determined horizontal crack, under the action of the Itype tensile force, the expansion of the crack from no to the crack expansion, and the corresponding crack expansion diagram, as shown in figure 17 below. 

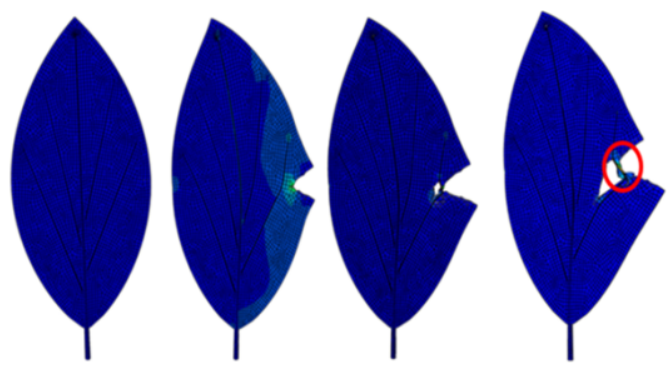

Figure 17. Grid and force diagram.

The total calculation time is $1 \mathrm{~s}$, and the whole fracture process and crack propagation from the force of the tobacco leaf to the tearing are analyzed. Before the crack meets the tobacco stem, the crack expands from the middle part of the tobacco leaf to the inside of the tobacco leaf according to the balance of forces. When the crack extends to the edge of the tobacco stem, the material and mechanical properties of the tobacco stem are completely different from the mesophyll. In terms of mechanical properties, the tobacco stem is much harder than the leaf flesh, and the overall force balance of the tobacco leaf is broken, and the tobacco stem is not easy to break. The inner leaf flesh part of the tobacco leaf connected with the tobacco stem will break when it reaches the strength limit, and the cracks will follow. The edge of the tobacco stem continues to expand.

It is assumed that the tobacco stems have a certain retarding effect on the crack growth of tobacco leaves. In order to verify this assumption, two parts are needed:

(1) Take the unit of one on the mesophyll crack and check the force change with time during the entire force process.

(2) Break and stretch the tobacco stems individually, select a unit at the break, and check the force change over time.

\section{Conclusion}

Through the simulation analysis of the I-shaped crack propagation of the whole tobacco leaf and the fracture tensile simulation analysis of the tobacco stem, the following conjectures are obtained.

(1) In the process of conducting I-mode fracture tensile simulation analysis on the whole tobacco leaf, as the tensile force increases, the length of the crack gradually increases. When the crack extends to the edge of the adjacent leaf vein, if it continues to expand in the direction of the crack, It needs more stretching force, more resistance, since the breaking force required to break the tobacco stem is much greater than the breaking force of the mesophyll, the crack will continue to propagate along the weaker side of the required breaking force, and obvious deflection will occur, so that the crack will move along the edge of the vein. Expand down. It is limitedly proved that the leaf veins have a good effect of retarding crack propagation. Or skip the tobacco stem to make the tobacco leaf on the other side crack.

Through mutual verification of the stress-strain curves obtained from the fracture tensile experiment of tobacco stem and the stress-strain curves obtained from the fracture tensile simulation of tobacco stem, the following conjectures were obtained.

(2) The trend of the stress-strain curve of tobacco stem fracture is basically similar to the stress-strain curve of experimental tobacco stem tensile fracture. Both have 
experienced the elastic stage, the plastic stage and the final damage stage, although the stress-strain curve above There are subtle differences between the elastic phase, the plastic phase, and the damage phase. In addition, the corresponding stress and strain values at the end of each phase are not the same. This is an inevitable error between the simulation results and the experimental results. The experimental results should prevail. However, the general curve trend and the experienced stage are basically unchanged, which indirectly verifies the simulation and experiment to each other, and initially completes the construction of the constitutive relationship of tobacco stem stretching.

But this article also has some shortcomings:

(1) Due to the experimental process of tobacco leaf mechanics, the temperature and humidity of the tobacco leaf itself have a great influence on its mechanical properties, and the parameters obtained from the experiment under different temperature and humidity conditions inevitably have errors.

(2) Due to the limitation of conditions, the experimental part is only the tensile fracture experiment. Although the model can be degraded and analyzed, it still has a certain impact on the accuracy of the finite element simulation.

\section{Acknowledgments}

This work is supported by China National Tobacco Corporation's major science and technology project "Research and application of "characterized and modular" threshing and redrying characteristics that highlight the tobacco leaf style in the producing area" (No.110201901031 (FK-02)), China National Tobacco Corporation Yunnan Branch Technology The planned key project "Research on the uniformity control of threshing and redrying slices" (No. 2018530000241024).

\section{References}

[1] WU Zuo-you,WEI Pan-pan, et al. Relationship between Physical Characteristics and Threshing Quality of Tobacco Leaf[J]. Acta Agriculturae Jiangxi,2014(26)

[2] National Tobacco Sales Administration. Cigarette Technology Specification[M]. Beijing: Central Literature Publishing House, 2003.

[3] Li Yuefeng, Jiang Huanyuan, et al. The relationship between tobacco leaf temperature and moisture content and threshing quality[J]. Tobacco Science and Technology 2005.

[4] Luo Fu Wei, et al. The influence of moisture and temperature on the quality of threshing leaves[J]. Machine China.

[5] Jiang Chengkang. Comprehensively promote the "high level of cigarettes" and strive to maintain the sustainable and healthy development of the industry [R], National Tobacco Work Conference Report, 2010 .

[6] Orowan E.Fracture and Strength of Solids.Reports on Progress in Phiysics,1948,XII:185.

[7] Evans, P.S. Leaf strength studies of pasture grasses.I.Apparatus, techniques and some factors affecting leaf strength[J].Journal of Agricultural Science,1967,69: 171-174.

[8] Daux C, Moes N, Dolbow J, Sukumar N, Belytschko T. Arbitrary Branched and Intersecting Cracks with the Extended Finite Element Method [J].International Journal for Numerical Methods in Engineering,2000,48:1741-1760.

[9] Sih G C. Handbook of stress-intensity factors[M]. Lehigh University, Institute of Fracture and Solid Mechanics, 1973.

[10] Rooke D P, Cartwright D J. Compendium of stress intensity factors[J]. Procurement Executive, Ministry of Defence. H. M. S. O. 1976, 330 p(Book)., 1976.

[11] Tada H, Prais P C, Irwin G T. The Stress Intensity Factor handbook[M].Hellertwon: Del Reasearch Corporation, 1985.

[12] Zhuang Zhuo, Liu Zhanli, Cheng Binbin, et al. Extended finite element method[M]. Beijing: Tsinghua University Press, 2012. 
[13] Yu Tiantang. Extended finite element analysis of discontinuity problems. Ship Mechanics. 2007, 11(5):716-722.

[14] Li Luxian, Wang Tiejun. Extended finite element (XFEM) and its application. Development of Mechanics. 2005, 35(1): 5-20

[15] Liu Changhong, Li Hongsheng, Peng Jun. Crack propagation analysis based on XFEM. Journal of Shanghai University of Engineering Science. 2010, 24(3): 218-220

[16] Griffith A A.The phenomena of rupture and flow in solids [J].Philosophical transactions of the royal society of London. Series A,containing papers of a mathematical or physical character,A1921,221:163198.

[17] Orowan E.Fracture and strength of solids [J]. Reports on Progress in Physics, 1949,12:185.

[18] Irwin G R.Fracture dynamics [J]. Fracturing of metals, 1948, 1948:147-196.

[19] Irwin G R. Analysis of stress and strains near the end of a crack traversing a plate [J]. Applied Mechanics, 1957, 24:109-114.

[20] Shen Chengkang. Fracture Mechanics[M]. Shanghai: Tongji University Press, 1996.

[21] Wright,W.,Vincent, J.F.V. Herbivory and the mechanics of fracture properties in plants[J]. Biological Reviews of the Cambridge Philosophical Society,1996, 71:401-413.

[22] Hong Qichao. Foundation of Engineering Fracture Mechanics[M]. Shanghai: Shanghai Jiaotong University Press, 1996. 